\title{
Incipient Slip and Long Range Plastic Strain Localization in Microtextured Ti-6Al-4V Titanium
}

\author{
McLean P. Echlin ${ }^{1, a}$, Jean Charles Stinville ${ }^{\mathrm{a}}$, Victoria M. Miller ${ }^{\mathrm{a}}$, William C. Lenthe ${ }^{\mathrm{a}}$, Tresa M. Pollock ${ }^{\mathrm{a}}$ \\ ${ }^{a}$ University of California Santa Barbara, Santa Barbara, California 93106-5050
}

\begin{abstract}
High resolution scanning electron microscope digital image correlation (SEM DIC) was performed in situ during uniaxial loading on Ti-6Al-4V rolled sheet titanium to determine the dependence of strain localization on microstructure and microtexture. Individual grains with preferred orientation for basal slip exhibited plastic localization along basal planes before macroscopic yielding. With additional strain, but still below macroscopic yielding, pyramidal and prismatic plastic activity was observed as slip bands transmitting across many grains and entire microtextured regions (MTRs). The localization of long range plastic strain occurred within MTRs that allowed for slip transmission across grains with low angle boundaries. The rolled sheet titanium material having strong [0001] and [1010] texture components showed pyramidal and prismatic type slip extending across entire MTRs at strains well below macroscopic yielding. These strain localization processes occur much earlier in straining and over lengthscales much longer than observed with conventional slip offset imaging. The implications for properties are discussed.
\end{abstract}

Keywords: titanium, scanning electron microscope digital image correlation, strain localization, microtextured regions, macrozones

\section{Introduction}

Ti-6Al-4V is the most common wrought titanium alloy, used in industries such as aerospace, biomedical, and chemical processing and synthesis. As a function of processing conditions, titanium alloys possess a wide range of microstructural conditions, which result in a wide range of material properties $[1,2]$. The processing history of a Ti alloy is therefore of critical importance to the development of the microstructure and texture present in a final component and has implications for design strategies, which increasingly can leverage anisotropic material properties [3]. An example of texture control in titanium was recently demonstrated using additive manufacturing methods [4]. After a homogenization step in the $\beta$ phase field, Ti-6Al$4 \mathrm{~V}$ alloy hot working in the $\alpha+\beta$ field can result in equiaxed $\alpha$ grain microstructure, for sufficiently slow cooling rates [2]. The resulting equiaxed microstructure is often forged, producing extensive mm-scale bands of grains with preferred grain orientation, known as microtextured regions (MTR)s or macrozones [3, 5-9], which subsequent deformation steps can enhance or break apart. The slip activity within the MTRs affects the mechanical properties of Ti-6Al-4V, including fatigue properties [6], through the localization of plastic deformation, motivating a more detailed understanding of the initial slip processes.

Slip activity in the hcp $\alpha$ phase has been studied for titanium alloys using high resolution SEM surface slip identification correlated with EBSD and Schmid factor (SF) analysis

\footnotetext{
次

* corresponding author

Email address: mechlin@engineering.ucsb.edu (McLean P. Echlin)
}

[10]. Pyramidal $\langle a\rangle$ and $\langle c+a\rangle$, prismatic, and basal type slip were all identified as active during room temperature tensile loading. The transmission of slip has been shown to be influenced by partial or full adherence to the Burgers orientation relation (BOR) between $\alpha$ grains separated by the $\beta$ phase [11]. However, the authors also note that dislocations still can crossslip and transmit through the $\beta$ phase. TEM analysis of titanium samples with a lath colony microstructure has revealed variability in dislocation transmission across the $\alpha-\beta$ boundary that is dependent on the presence of residual dislocations at the boundary, even when satisfying the BOR [12]. SEM-based surface slip trace analysis in strained samples showed a dependence on slip transmission between $\alpha$ and $\beta$ requiring aligned slip planes with high Schmid factors for both phases [13, 14]. Most current research utilizes SEM-based surface slip trace analysis to determine slip activity, which is dependent on the extrusion and/or intrusion of slip bands at the free surface. This approach has been shown to provide incomplete information on the operative slip processes [15, 16]. Recently, resolution improvements in scanning electron microscope digital image correlation (SEM DIC) techniques [15-19] allow measurement of in-plane plastic strain distribution at the sub-micrometer scale, which is not resolved by conventional SEM imaging. In this study, in situ tensile loading of Ti-6Al-4V specimens was performed to determine the slip system activity by high resolution SEM DIC. The extent of strain localization, the relation of the localization to the microstructure, and the relative activity of slip systems is described in the following sections. 


\section{Experimental}

Commercially available mill-annealed Titanium Ti-6V-4Al alloy rolled plate fabricated by Timet was investigated. The $\alpha-\beta$ equiaxed microstructural condition was subjected to a heat treatment at $926^{\circ} \mathrm{C}$ for 4 hours followed by a $30^{\circ} \mathrm{C} /$ hour slow cool to homogenize. SEM and EBSD maps of the equiaxed microstructure are shown in Figure 1(a-c). Tensile specimens were electrodischarge machined (EDM) from the plate material into flat dogbone samples of dimensions $11 \mathrm{~mm} \times 52 \mathrm{~mm} \times$ $1 \mathrm{~mm}$, with a gauge cross-section of $1 \mathrm{~mm}$ by $3 \mathrm{~mm}$ and length of $10 \mathrm{~mm}$. All surfaces of the samples were metallographically prepared with $\mathrm{SiC}$ to 1200 grit. Additionally, one of the $11 \mathrm{~mm}$ $\mathrm{x} 52 \mathrm{~mm}$ faces was polished with a $6 \mu \mathrm{m}$ diamond suspension and then chemo-mechanically polished in colloidal silica for 12 hours.

Electron backscatter diffraction (EBSD) maps were collected from $2 \mathrm{~mm} \times 2 \mathrm{~mm}$ areas on the polished surface with $0.55 \mu \mathrm{m} /$ pixel resolution with an EDAX Hikari XP detector, as shown in Figure 1(a). MTRs with different orientation are observed with $100-400 \mu \mathrm{m}$ size along the ND direction that extend for millimeters along the TD and RD directions. The electron beam conditions used for EBSD mapping at $70^{\circ}$ tilt before and after deforming the sample were a $30 \mathrm{kV}$ beam energy, $6.55 \mathrm{nA}$ beam current, with a $40 \mu \mathrm{m}$ SEM aperture. The EBSD detector was operated using a $4 \times 4$ camera binning mode with a 200 frames per second collection speed. A grain dilation routine was applied to the EBSD data to remove misindexed points, primarily at or near the grain boundaries, for grains smaller than 5 pixels. Each grain, as determined by EBSD with a $5^{\circ}$ tolerance, was also assigned an average orientation and used for the calculation of slip activity. Texture analysis using a harmonic series expansion with a series rank of 31 was performed and is represented in the inverse pole figure and pole figure plots.

Secondary electron SEM images collected with the electron beam normal to the surface $\left(0^{\circ}\right.$ tilt $)$ were made from selected areas in Figure 1(b) within the EBSD mapped regions in Figure 1(a). The field of view (FOV) in Figure 1(b) is composed of many high resolution (6144 x 4096) images, one of which is shown in Figure 1(c). The entire gauge section of the sample was speckle patterned for digital image correlation (DIC) measurements by chemically etching using Kroll's solution at room temperature for 15 seconds. The alpha phase was preferentially etched, exposing 1-10 $\mu \mathrm{m}$ elevated regions of beta phase. The speckle pattern in Figure 1(d) is composed of circular particles with diameter ranging between 50-250 nm with similar spacing between particles. This speckle pattern allows for in situ SEM DIC and simultaneous acquisition of EBSD information from the sample surface. The specific DIC parameters used to measure strain are discussed in Section 2.1. After DIC measurements were performed on the loaded samples, EBSD maps were again collected from the DIC measurement regions of interest. These EBSD maps were collected with the same parameters as described previously and were carefully aligned to the SEM DIC strain maps using affine transformation and alignment routines originally developed for merging 3-D multimodal datasets [20].

\subsection{Digital Image Correlation}

DIC measurements on the etched Ti-6Al-4V samples permitted examination of the dependence of initial straining on microstructural features and their relationship to the subsequent localization of strain within the microstructure. The speckle pattern produced by chemical etching generated random particle sizes and shapes, though mostly equiaxed, ranging from 50$250 \mathrm{~nm}$ as shown in Figure 1(d). The speckle pattern is much finer than the grain size of both the $\alpha$ and $\beta$ phases, which have average sizes of $18.5 \mu \mathrm{m}$ and $2.5 \mu \mathrm{m}$, respectively, weighted by area fraction. Considerations for the total area analyzed with the SEM DIC technique were made based on the $\alpha$ and $\beta$ phase grain sizes as well as the MTR sizes in order to capture strain localization effects at both lengthscales, as detailed elsewhere [21]. However, subgrain scale SEM DIC resolution must also be considered in order to measure strain localization at the slip band scale. The DIC parameters included a subset size of 21 by 21 pixels $(0.472$ by $0.472 \mu \mathrm{m})$ with a step size of 3 pixels $(0.067$ $\mu \mathrm{m})$. The parameters were selected to limit SEM distortions while maximizing the horizontal field width (HFW), discussed in more detail elsewhere for SEM DIC [17, 22], with conditions shown to be optimal in prior work [16, 23]. All DIC calculations were performed with Vic2D 2009 [24], using an image mask to remove the beta phase regions from the SEM image data. The SEM DIC technique coupled with EBSD information allows for direct comparison with results obtained from crystal plasticity simulations.

\subsection{Mechanical Test}

Mechanical testing was performed both ex situ and in situ to determine the macroscopic deformation response and the local strain states on the sample surface, respectively. All mechanical tests were performed at room temperature with strain rates of $10^{-3}$ to $10^{-4}$ on polished dogbone type samples with a $1 \times 3 \mathrm{~mm}$ gauge section. An MTS instrument was used for macroscopic tensile testing in load control using an extensometer to measure displacement in the gauge section. In situ DIC experiments utilized a mechanical loading cell that was inserted into the SEM. Macroscopic strain was tracked by measuring displacements between fiducial marks on the sample surface in the SEM and also with an extensometer type strain gauge, which was monitored during loading. The stress-strain response of the Ti-6Al-4V alloy uniaxially loaded along the sample rolling direction (RD) is shown in Figure 2. SEM DIC strain measurements were performed at macroscopic strains of $0.65,0.71$, and $0.86 \%$, for samples that were loaded along the RD, as indicated on the stress-strain plot in Figure 2. Samples were also loaded along the transverse rolling direction (TD) with SEM DIC measurements made at macroscopic strains of $0.58,0.77$, and $1.0 \%$. The SEM DIC strain measurements collected at these macroscopic strains will be presented in Section 3.

\section{Results}

The extensive long range strain localization observed in this Ti-6Al-4V alloy required that large fields of view $(500 \mu m \mathrm{x}$ 
$500 \mu \mathrm{m})$ were collected at high spatial resolutions $(0.067 \mu \mathrm{m}$ step size and 0.472 by $0.472 \mu \mathrm{m}$ subset size) with precise alignment of the imaging modalities (EBSD, secondary electron $(\mathrm{SE})$ ) in order to relate the strain localization to the microstructure.

\subsection{Microstructure and Mechanical Response}

To assess the anisotropy of the mechanical response due to the rolling process, regions were investigated from surfaces with the RD-ND and TD-ND directions in the imaging plane. The inverse pole figure (IPF) maps in Figure 3 and Figure 4 show the grain orientations with respect to the loading direction, which is parallel to RD or TD, respectively. The regions investigated show strong texture along the [0001] and [1010] directions with respect to the sample $\mathrm{RD}$, with maximum values of the orientation distribution function up to 20 times random. For the samples imaged in the RD-ND plane and the TD-ND plane, Figure 3 and Figure 4, a large fraction of the grains in the MTRs have orientations near the [0001] or [1010]. Three specific MTRs were studied, as shown in Figure 3, one having predominant orientation near the [1010] (labeled as MTR 1), another having orientation near the [0001] (labeled as MTR 2), and a region with a more random orientation distribution (labeled as MTR 3).

Bulk Ti-6Al-4V samples were uniaxially strained in tension along the RD direction to produce the stress-strain plot shown in Figure 2. The elastic modulus of the material loading along the sample RD was $116 \mathrm{GPa}$ and had a $0.2 \%$ yield stress of $915 \mathrm{MPa}$. Flat specimens were machined, polished and etched from the same Ti-6Al-4V material and again uniaxially strained in tension along the specimen RD to the macroscopic strains shown in Figure 2:(a) 0.65, (b) 0.71, and (c) 0.86\%, where SEM DIC measurements were made.

\subsection{Strain Localization at the Surface}

Strain localization was characterized using SEM DIC on surfaces of samples with RD/ND and TD/ND in the imaging plane, shown in Figure 5 and Figure 6, respectively. The regions were selected because they contained combined sub-regions with microtexture (MTRs) and with clusters of semi-random grain orientations.

At relatively low strains such as $0.65 \%$, shown in Figure 5, strain localized along individual slip planes within individual $\alpha$ grains. However, upon further loading the regions of strain localization extended through the neighboring $\alpha$ grains, in many cases across entire MTRs. The extension of strain localization across microstructural boundaries is even more pronounced in the shear strain $\left(\epsilon_{x y}\right)$ map shown in Figure 7 . The length of localized bands were measured and are plotted as a function of the applied global strain in Figure 8 showing that slip bands and strain localizations exist across as many as 21 grains, well below the macroscopic onset of plasticity (yield strength) at 0.71 and $0.86 \%$ strain.

Furthermore, bands of strain localization are shown to transmit through the $\beta$ grains, the dark regions in the strain maps in Figure $9(\mathrm{~d}, \mathrm{f}, \mathrm{h})$, between the $\alpha$ grains. Only at strains beyond $1.0 \%$ do slip traces (shear or extrusions) begin to become visible in the high resolution secondary electron SEM images without using DIC, Figure 9(a,c,e,g,i). Out of plane extrusions do correlate with slip band formation on the surface of material at large macroscopic strains, imaged by secondary electron SEM, however in-plane shearing within grains is not resolved, as demonstrated by a comparison of Figure 9(c and g) to Figure 9(f and h). The images in Figure 10(c) show in-plane strain measured using the secondary SEM images for unstrained (Figure 10(a)) and 1.0\% strained (Figure 10(b)) Ti-6Al-4V specimen. Incipient slip, as is apparent from Figure 10(b) is not easily observable without DIC analysis. Readers may wish to refer to the associated movie in the Supplemental Material, where slip bands are shown to extend up to a $\beta$ grain as observed in the micrograph macroscopically strained to $1.0 \%$ in Figure 10(e). However, transmission across the $\beta$ grain into the adjacent $\alpha$ grain is only resolved in the SEM DIC strain measurement, shown in the inset region of Figure $9(\mathrm{f}, \mathrm{h})$, and not in the secondary electron SEM image. The strain localization events connect the $\alpha$ grains with different active slip systems despite the presence of $\beta$ grains, which will be discussed in detail presently. The movies provided in the Supplemental Material, which refer to Figure 10(d and e), indicate significant shearing in the $\beta$ grain.

The SEM DIC strain measurements were aligned with the EBSD maps in order to determine types of active slip systems on a grain-by-grain basis and to compare with the location of the MTRs. The type of slip system activity in each grain was determined by comparing the angle of the localization band measured using SEM DIC to the angle of the calculated slip trace on the surface, resolved using the EBSD grain orientation information, as plotted in Figure 11 and Figure 12 for the measurements in the RD-ND and TD-ND planes. A small proportion of the grains $(<9 \%)$ had surface slip traces that were indistinguishable between the pyramidal and prismatic slip systems and were excluded from the analysis. At large deformations however, the ability to discern the independent slip system type may become challenging due to grain rotations, relative order of slip system activation, and microscope distortions. Especially at large macroscopic strains, emerging methods for the identification of slip systems [25] may become necessary.

The orientations of the grains that exhibited strain localization as a result of basal, prismatic, and pyramidal type slip are plotted with the calculated Schmid Factor (SF) contours on the inverse pole figures at each strain increment for both sample orientations, shown in Figure 11 and Figure 12. Only the pyramidal $\langle a\rangle$ type SF is shown on the contour plots; however $\langle c+a\rangle$ type is considered in more detail in Figure 13. The orientations of grains plotted for each strain increment correspond to additional localization events since the previous strain increment.

Initially at low strains of $0.65 \%$ and $0.55 \%$, the grains oriented such that they have a high SF for basal slip exhibit early strain localization, shown as the blue points in Figure 11 and Figure 12. However, with increased strains of $0.71 \%$ and $0.77 \%$, the proportion of grains oriented with high SFs for both prismatic slip and pyramidal slip become greater, as evident by the grain orientations marked with red (prismatic) and green (pyramidal) points which show non-basal shear localization. 
The grains observed to have pyramidal slip have a Burgers vector of either $\langle a\rangle$ or $\langle c+a\rangle$ type. The large number of grains with orientations near the [0001] such as those in the MTR region labeled 2, also shown by the pole figures (PF) in Figure 3 and Figure 4, preferentially exhibit pyramidal type slip. These grains have a high SF for pyramidal slip $\langle c+a\rangle$ type, when compared to pyramidal slip $\langle a\rangle$ type SFs for near [0001] orientations. The slip system activity of a near [0001] grain is shown in detail in Figure 13, where the surface slip traces are calculated for each possible slip system. The strain map in Figure 13 shows that the direction of the slip trace closely matches 1 st and 2 nd order $\langle c+a\rangle$ type pyramidal slip traces and $\langle a\rangle$ type pyramidal slip. The maximum SF for both 1 st and 2nd order $\langle c+a\rangle$ type slip are greater than 0.45 for near [0001] orientations, whereas the SF for $\langle a\rangle$ type pyramidal slip is below 0.2. MTRs with orientation near [1010] such as those labeled 1, predominately show prismatic type slip. The MTRs with more random orientations, such as those labeled 3, show activation of all three slip systems with some bias toward the basal slip.

All SEM DIC strain measurements were performed below macroscopic yielding in the Ti-6Al-4V samples. Localized plasticity is observed by SEM DIC as shown in Figure 5 and Figure 6, which is associated with slip activity. Additionally, elastic strains can also be resolved in the SEM DIC measurements. In reference to the horizontal loading direction the elastic modulus of individual grains has been calculated, Figure 14(b). The high modulus regions (i.e. MTR 2) arise from the texturing.

Elastic strains measured below $0.8 \%$ require different visualization parameters than those required for plasticity. The $\epsilon_{x x}$ strain field has been measured at $0.71 \%$ macroscopic strain in Figure 14 which includes both the elastic and plastic strain fields. An average variation in strain can be observed between the MTRs labeled 1 and 2, as a result of elastic strain contributions. The [1010] oriented grains (labeled MTR 1 - blue in the IPF map) have higher average $\epsilon_{x x}$ total strain per grain, by roughly $0.15 \%$, in relation to the [0001] oriented grains (labeled MTR 2 - red in the IPF map). The spatial relation of the diffuse elastic strain with respect to the MTRs is shown in Figure 14, where the elastic modulus, (b), of the average orientation of the grains is shown in comparison to a $\epsilon_{x x}$ strain map, (c), for the same microstructural region at $0.71 \%$ macroscopic tensile strain. The MTR labeled 2 has high elastic modulus and shows low elastic strains, compared with the MTR labeled 1, which shows the opposite trend.

\section{Discussion}

The SEM DIC strain measurements show extensive localized plastic straining below the $0.2 \%$ yield strength of the material, as shown in Figure 5 and Figure 6. Comparison of the calculated surface slip trace based on the grain orientations to the measured strain localization on the surface indicates the type of slip activity present up to the onset of macroscopic yielding, in Figure 11 and Figure 12. In the rolled equiaxed Ti-6Al-4V material tested here, basal type oriented grains preferentially exhibited strain localization upon loading. These basal orientated grains and the basal slip they produce is isolated from the surrounding MTRs, as shown by the strain localization within single grains in the lowest macroscopic strain measurements at $0.65 \%$ and $0.58 \%$, for samples loaded in tension along the $\mathrm{RD}$ and TD, respectively. With continued loading, but still well below macroscopic yielding, pyramidal and prismatic slip develop with long range localization across MTRs containing grains with similar orientations. The material tested has strong [0001] and [1010] texture components, shown in Figure 3 and Figure 4, which predisposes the material for early pyramidal type slip. The SF for 1 st and 2 nd order $\langle c+a\rangle$ type slip is near 0.5 for [0001] grain orientations, however $\langle a\rangle$ type pyramidal slip also has a high SF compared to prismatic and basal near the [0001]. The surface slip trace analysis employed here cannot distinguish between 1 st and 2 nd order $\langle c+a\rangle$ and $\langle a\rangle$ pyramidal type slip.

Individual MTRs were observed to have differing mechanical response, depending on the net orientation of the MTR. MTRs with primarily [1010] orientation and labeled as MTR 1 in the IPF maps show mostly prismatic type slip, while the [0001] orientation MTRs labeled as MTR 2 show mostly pyramidal. The regions with more random orientation, labeled MTR 3, show contributions from of all three slip systems with some bias toward the basal slip.

In addition to the SFs of each slip system, the relative critical resolved shear stress (CRSS) values will play a role in determining which slip systems are active. Reported CRSS values for all slip modes from the literature [10, 26-33] are shown in Table 1. Pyramidal $\langle c+a\rangle$ slip in titanium alloys is most commonly reported to be 1st order [26], though in many cases the Burgers vector is determined but the plane is not confirmed. Generally, all three modes of $\langle a\rangle$-type slip are soft relative to $\langle c+a\rangle$ slip, with the prismatic $\langle a\rangle$ to pyramidal $\langle c+a\rangle$ CRSS ratio falling between 1:1.6 and 1:3.9 for the considered alloys. Of the $\langle a\rangle$-type slip modes, prismatic is typically reported to be softest, with basal and pyramidal $\langle a\rangle$ slip typically being only slightly harder. The CRSS ratio between pyramidal $\langle c+a\rangle$ and pyramidal $\langle a\rangle$ slip is nearly the inverse of the ratio between the SFs, indicating that either type of slip may be occurring in the [0001]-oriented grains.

At total strains between $0.7 \%-0.9 \%$, strain localization continues with the longest band spanning across 21 grains (258 $\mu \mathrm{m}$ ), as shown in Figure 5 and Figure 6. Long range strain localization was even observed for many non-basal slip configurations, as enabled by the banded MTRs containing many grains with similar orientations. The MTRs are 100-400 $\mu \mathrm{m}$ measured along the ND direction, while extending millimeters in the TD or RD directions. In other engineering materials such as polycrystalline superalloys and stainless steel, slip is localized within 2-4 grains (roughly $88 \mu \mathrm{m}$ ) [16] and 2-4 grains (roughly $100 \mu \mathrm{m}$ ) [18], when loaded to strains near the onset of yielding. Furthermore, long bands of shear $\left(\epsilon_{x y}\right)$ are also shown to develop in MTRs at strains between $0.7 \%-0.9 \%$, with slip transmission between grains of similar orientations. The length of the concentrated shear band is dependent on the orientation of the MTR and the ability of slip to transmit between grains of 
similar orientation. This enables the propagation of slip across a MTR, based on the preferred orientation of the region. In addition to the plastic localization processes observed here, the overall heterogeneity of the straning process is also influenced by the elastic properties of the material.

The elastic anisotropy of the hcp $\alpha$ phase [34, 35] is apparent in Figure 14(b), with high contrast between the MTR regions that predominately have high or low elastic modulus. The average strain in the MTRs scales with the elastic modulus as shown in the strain measurements for the same field of view in Figure 14(c). Within MTR 2 a small number of grains exhibit orientations that are far from the predominant basal orientation of the MTR. They are spatially located within the MTR and are constrained by the deformation of the MTR and therefore are more likely to develop plasticity. For example, the grains in MTR 2 that show strain localization first (non-red in IPF) are softer oriented and constrained by the predominantly basal oriented grains (red in IPF) that are deforming in unison, resulting in enhanced plasticity in the former. Furthermore, grains that had localized strain at small deformation initiated long range plastic strain localization in nearby grains that extended across entire MTRs having implications for crack initiation and fatigue. Basal oriented MTRs that contain grains with dissimilar orientations (such as MTR 2), and high elastic anisotropy, have been shown to initiate cracks during low cycle fatigue [6]. Also in low cycle fatigue, a high frequency of crack initiation events and subsequent coalescence has been observed in basal and prismatic MTRs, but not in orientations that are less favorably oriented for slip [36]. Furthermore, at larger lengthscales, the preferential grain orientation distribution intrinsic to MTRs arranges the structure of rolled components such that adjacent MTRs will also be strained non-uniformly.

\section{Conclusions}

The experiments performed using SEM DIC provide evidence that long range strain localization occurs across MTRs at strains below macroscopic yield conditions. The relationship between the orientation of the MTR and the loading condition determines the activity of the slip system type, however pyramidal and prismatic slip are easily activated in these rolled materials due to preferential [0001] and [1010] texture. Pyramidal slip was observed in [0001] oriented MTRs, likely to be $\langle c+a\rangle$ type because of high SF for these grains. The bands of localized strain developed via prismatic slip spanning as many as 21 grains $(258 \mu \mathrm{m})$, and have been shown to originate from some grains showing early basal type slip in MTRs with [0001] grain orientations. In fatigue conditions where the applied macroscopic strain is often below the yield strength, the basal oriented grains will exhibit slip band localization in the first cycle. Basal slip was observed to initiate at the lowest strains, however the long range strain localization at elevated strain (still below macroscopic yielding) was primarily pyramidal and prismatic type. Furthermore, elastic anisotropy influenced the overall distribution of strain within MTRs with different preferred orientations.

\section{Acknowledgments}

The authors thank Professor Paul Dawson for useful discussions on titanium micromechanics and Remco Geurts for microscope automation expertise. We also acknowledge our funding sources, the AFRL Center of Excellence grant (FA9550-121-0445) and ONR grants (N00014-12-1-0039 and N00014-121-0075).

\section{References}

[1] D. Banerjee, J. Williams, Microstructure and slip character in titanium alloys, Defence Science Journal 36 (2) (1986) 191-206.

[2] D. Banerjee, J. C. Williams, Perspectives on titanium science and technology, Acta Materialia 61 (3) (2013) 844 - 879, the Diamond Jubilee Issue Materials Challenges in Tomorrow's World Selected Topics in Materials Science and Engineering. doi:http://dx.doi.org/10.1016/j.actamat.2012.10.043.

[3] M. G. Glavicic, V. Venkatesh, Integrated computational materials engineering of titanium: Current capabilities being developed under the metals affordability initiative, JOM 66 (7) (2014) 1310-1320. doi:10.1007/s11837-014-1013-0.

[4] R. R. Dehoff, M. M. Kirka, W. J. Sames, H. Bilheux, A. S. Trem$\sin$, L. E. Lowe, S. S. Babu, Site specific control of crystallographic grain orientation through electron beam additive manufacturing, Materials Science and Technology 31 (8) (2015) 931-938. doi:10.1179/1743284714Y.0000000734.

[5] A. P. Woodfield, M. D. Gorman, R. R. Corderman, J. A. Sutliff, B. Yamrom, Titanium '95: science and technology, in: P. Blenkinsop, W. Evans, H. Flower (Eds.), proceedings of the Eighth World Conference on Titanium, Institute of Materials, 1995.

[6] F. Bridier, P. Villechaise, J. Mendez, Slip and fatigue crack formation processes in an $\alpha / \beta$ titanium alloy in relation to crystallographic texture on different scales, Acta Materialia 56 (15) (2008) 3951 - 3962. doi:http://dx.doi.org/10.1016/j.actamat.2008.04.036.

[7] L. Germain, N. Gey, M. Humbert, P. Vo, M. Jahazi, P. Bocher, Texture heterogeneities induced by subtransus processing of near titanium alloys, Acta Materialia 56 (16) (2008) 4298 - 4308. doi:http://dx.doi.org/10.1016/j.actamat.2008.04.065.

[8] A. L. Pilchak, C. J. Szczepanski, J. A. Shaffer, A. A. Salem, S. L. Semiatin, Characterization of microstructure, texture, and microtexture in near-alpha titanium mill products, Metallurgical and Materials Transactions A 44 (11) (2013) 4881-4890. doi:10.1007/s11661-013-1804-x.

[9] J. C. Stinville, F. Bridier, D. Ponsen, P. Wanjara, P. Bocher, High and low cycle fatigue behavior of linear friction welded Ti-6Al$4 \mathrm{~V}$, International Journal of Fatigue 70 (0) (2015) 278 - 288. doi:http://dx.doi.org/10.1016/j.ijfatigue.2014.10.002.

[10] F. Bridier, P. Villechaise, J. Mendez, Analysis of the different slip systems activated by tension in a $\alpha / \beta$ titanium alloy in relation with local crystallographic orientation, Acta Materialia 53 (3) (2005) 555 - 567. doi:http://dx.doi.org/10.1016/j.actamat.2004.09.040.

[11] D. He, J. Zhu, S. Zaefferer, D. Raabe, Effect of retained beta layer on slip transmission in $\mathrm{Ti}-6 \mathrm{Al}-2 \mathrm{Zr}-1 \mathrm{Mo}-1 \mathrm{~V}$ near alpha titanium alloy during tensile deformation at room temperature, Materials \& Design 56 (2014) 937 - 942. doi:http://dx.doi.org/10.1016/j.matdes.2013.12.018.

[12] S. Suri, G. B. Viswanathan, T. Neeraj, D. H. Hou, M. J. Mills, Room temperature deformation and mechanisms of slip transmission in oriented single-colony crystals of an / titanium alloy, Acta Materialia 47 (3) (1999) 
1019 - 1034. doi:http://dx.doi.org/10.1016/S1359-6454(98)00364-4.

[13] J. R. Seal, M. A. Crimp, T. R. Bieler, C. J. Boehlert, Analysis of slip transfer and deformation behavior across the $\alpha / \beta$ interface in Ti-5 $\mathrm{Al}-2.5 \mathrm{Sn}$ (wt.\%) with an equiaxed microstructure, Materials Science and Engineering: A 552 (2012) 61 - 68. doi:http://dx.doi.org/10.1016/j.msea.2012.04.114.

[14] Y. Guo, T. B. Britton, A. J. Wilkinson, Slip band-grain boundary interactions in commercial-purity titanium, Acta Materialia 76 (0) (2014) 1 - 12. doi:http://dx.doi.org/10.1016/j.actamat.2014.05.015.

[15] J. C. Stinville, M. P. Echlin, D. Texier, F. Bridier, P. Bocher, T. M. Pollock, Sub-grain scale digital image correlation by electron microscopy for polycrystalline materials during elastic and plastic deformation, Experimental Mechanics (2015) 1-20. doi:10.1007/s11340-015-0083-4.

[16] J. C. Stinville, N. Vanderesse, F. Bridier, P. Bocher, T. M. Pollock, High resolution mapping of strain localization near twin boundaries in a nickel-based superalloy, Acta Materialia 98 (2015) 29 - 42. doi:http://dx.doi.org/10.1016/j.actamat.2015.07.016.

[17] A. D. Kammers, S. Daly, Digital image correlation under scanning electron microscopy: Methodology and validation, Experimental Mechanics 53 (9) (2013) 1743-1761. doi:10.1007/s11340-013-9782-x.

[18] F. Di Gioacchino, J. Quinta da Fonseca, Plastic strain mapping with sub-micron resolution using digital image correlation, Experimental Mechanics 53 (5) (2013) 743-754.

[19] L. Patriarca, W. Abuzaid, H. Sehitoglu, H. J. Maier, Slip transmission in bcc fecr polycrystal, Materials Science and Engineering: A 588 (0) (2013) 308 - 317. doi:http://dx.doi.org/10.1016/j.msea.2013.08.050.

[20] W. C. Lenthe, M. P. Echlin, A. Trenkle, M. Syha, P. Gumbsch, T. M. Pollock, Quantitative voxel-to-voxel comparison of TriBeam and DCT strontium titanate three-dimensional data sets, Journal of Applied Crystallography 48 (4). doi:10.1107/S1600576715009231.

[21] C. Efstathiou, H. Sehitoglu, J. Lambros, Multiscale strain measurements of plastically deforming polycrystalline titanium: Role of deformation heterogeneities, International Journal of Plasticity 26 (1) (2010) 93 106. doi:http://dx.doi.org/10.1016/j.jplas.2009.04.006.

[22] A. D. Kammers, S. Daly, Small-scale patterning methods for digital image correlation under scanning electron microscopy, Measurement Science and Technology 22 (12) (2011) 125501.

[23] J. Stinville, W. Lenthe, J. Miao, T. Pollock, A combined grain scale elastic-plastic criterion for identification of fatigue crack initiation sites in a twin containing polycrystalline nickel-base superalloy, Acta Materialia 103 (2016) 461 - 473. doi:http://dx.doi.org/10.1016/j.actamat.2015.09.050.

[24] Vic-2D (2009) [software], Correlated Solutions Inc., Columbia, SC.

[25] Z. Chen, S. H. Daly, Active slip system identification in polycrystalline metals by digital image correlation (dic), Experimental Mechanics (Under review).

[26] I. P. Jones, W. B. Hutchinson, Stress-state dependence of slip in titanium6Al-4V and other HCP metals, Acta Metallurgica 29 (1981) 951-968.

[27] J. C. Williams, R. G. Baggerly, N. E. Paton, Deformation behavior of HCP Ti-Al alloy single crystals, Metallurgical and Materials Transactions A 33 (13) (2002) 837-850.

[28] I. Bantounas, D. Dye, T. C. Lindley, The effect of grain orientation on fracture morphology during high-cycle fatigue of Ti-6Al-4V, Acta Materialia 57 (12) (2009) 3584-3595. doi:10.1016/j.actamat.2009.04.018.
[29] V. Hasija, S. Ghosh, M. J. Mills, D. S. Joseph, Deformation and creep modeling in polycrystalline Ti-6Al alloys, Acta Materialia 51 (15) (2003) 4533-4549. doi:10.1016/S1359-6454(03)00289-1.

[30] F. Bridier, D. L. McDowell, P. Villechaise, J. Mendez, Crystal plasticity modeling of slip activity in Ti-6Al-4V under high cycle fatigue loading, International Journal of Plasticity 25 (6) (2009) 1066-1082. doi:10.1016/j.ijplas.2008.08.004.

[31] M. Knezevic, R. A. Lebensohn, O. Cazacu, B. Revil-Baudard, G. Proust, S. C. Vogel, M. E. Nixon, Modeling bending of -titanium with embedded polycrystal plasticity in implicit finite elements, Materials Science and Engineering: A 564 (2013) 116-126. doi:10.1016/j.msea.2012.11.037.

[32] G. Venkataramani, D. Deka, S. Ghosh, Crystal plasticity based FE model for understanding microstructural effects on creep and dwell fatigue in Ti-6242, Journal of Engineering Materials and Technology 128 (3) (2006) 356. doi:10.1115/1.2204942.

[33] W. B. Hutchinson, M. R. Barnett, Effective values of critical resolved shear stress for slip in polycrystalline magnesium and other hcp metals, Scripta Materialia 63 (7) (2010) 737-740. doi:10.1016/j.scriptamat.2010.05.047.

[34] D. Tromans, Elastic anisotropy of hep metal crystals and polycrystals, Int. J. Res. Rev. Appl. Sci 6 (4) (2011) 462-483.

[35] N. R. Barton, P. R. Dawson, On the spatial arrangement of lattice orientations in hot-rolled multiphase titanium, Modelling and Simulation in Materials Science and Engineering 9 (5) (2001) 433.

[36] K. Le Biavant, S. Pommier, C. Prioul, Local texture and fatigue crack initiation in a ti-6al-4v titanium alloy, Fatigue \& Fracture of Engineering Materials \& Structures 25 (6) (2002) 527-545. doi:10.1046/j.14602695.2002.00480.x. 
Table 1: CRSS ratios reported in literature. Pyramidal $\langle c+a\rangle$ either refers to first order or where the slip plane was not determined. Table modeled after Ref. [33].

\begin{tabular}{lccccccccc}
\hline Material & \multicolumn{3}{c}{ Critical Resolved Shear Stresses (MPa) } & \multicolumn{5}{c}{ CRSS Normalized to Prismatic } & Ref. \\
& Basal & Prism. & Pyr. $\langle a\rangle$ & Pyr. $\langle c+a\rangle$ & Basal & Prism. & Pyr. $\langle a\rangle$ & Pyr. $\langle c+a\rangle$ & \\
\hline Ti-6Al-4V & 444 & 392 & 404 & 631 & 1.1 & 1.0 & 1.1 & 1.6 & {$[26]$} \\
Ti-6Al & 190 & 200 & & 770 & 1.0 & 1.0 & & 3.9 & {$[27]$} \\
Ti-6Al-4V & & & & & 1.0 & 1.0 & & 2.6 & {$[28]$} \\
Ti-6Al & 322 & 320 & & 846 & 1.0 & 1.0 & & 2.6 & {$[29]$} \\
Ti-6Al-4V & 420 & 370 & 490 & 590 & 1.1 & 1.0 & 1.3 & 1.6 & {$[30]$} \\
Ti-6Al-4V & 388 & 373 & & & 1.0 & 1.0 & & & {$[10]$} \\
CP Ti & & 98 & & 224 & & 1.0 & & 2.3 & {$[31]$} \\
Ti-6242 & 365 & 345 & & 620 & 1.1 & 1.0 & & 1.8 & {$[32]$} \\
\hline
\end{tabular}



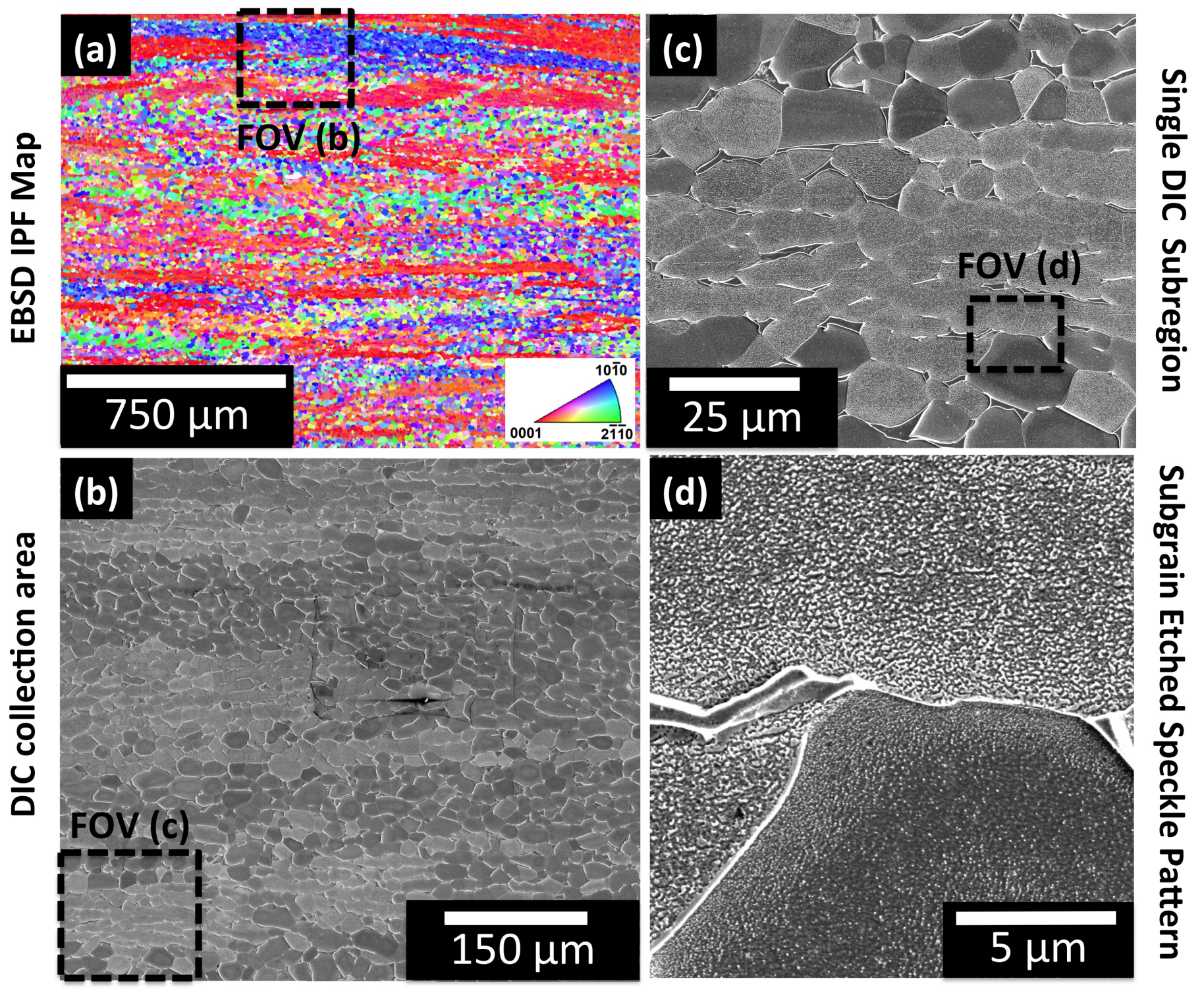

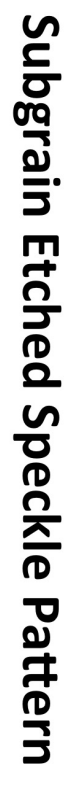

Figure 1: Ti-6Al-4V alloy was investigated in the mill annealed $\alpha-\beta$ condition, which is primarily composed of equiaxed $\alpha$ grains that are strongly textured from rolling steps in the $\alpha-\beta$ phase field. Microtextured regions (MTRs) are present at the scale of $100 \mu m$-mm, as shown in (a). (b) Selected field of view (FOVs) were analyzed with subgrain resolution SEM digital image correlation (DIC), which exhibited MTRs of varied orientations and semi-randomly textured regions. The FOV in (b) is composed of many high resolution secondary electron SEM images which resolve the speckle pattern, (d), which has been etched into the microstructure as discussed in Section 2. 


\section{Ti-6AI4V - RD Loading Direction}

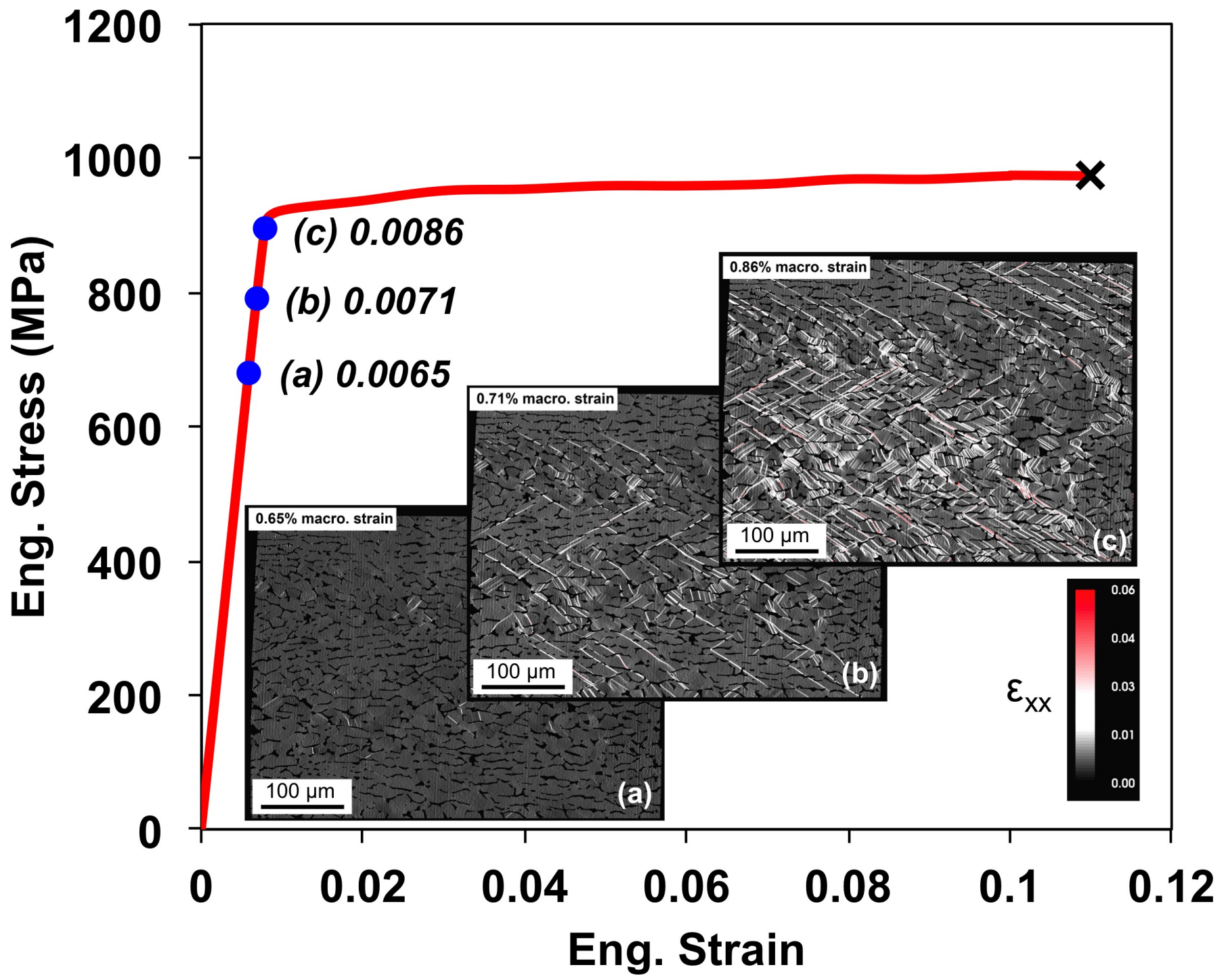

Figure 2: Mechanical testing of Ti-6Al-4V performed at room temperature loaded uniaxially and quasistatically resulted in the global stress-strain response for a sample with the loading direction oriented along the sample rolling direction (RD). SEM DIC measurements were made at the following strains: (a) 0.0065, (b) 0.0071 , and (c) 0.0086 for the RD parallel to the loading direction samples, and at $0.0058,0.0077$, and 0.01 for samples with the loading direction parallel to the transverse rolling direction (TD). 


\section{IPF map - RD Reference}
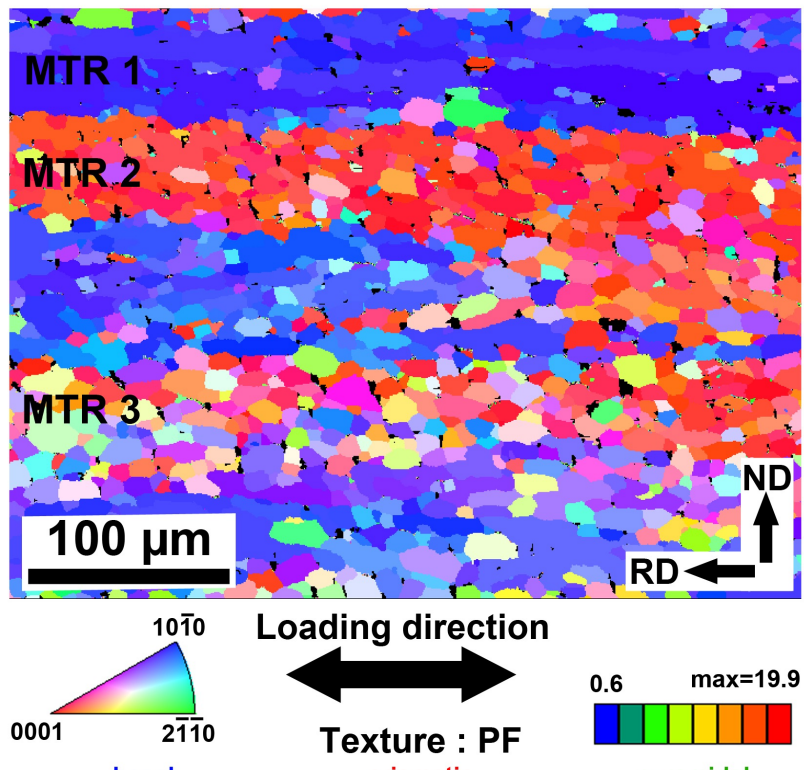

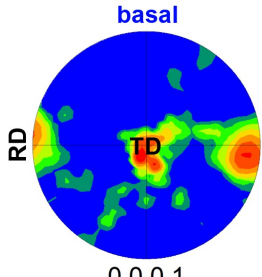

0001

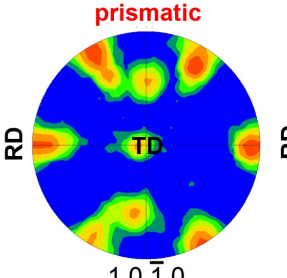

$10 \overline{1} 0$

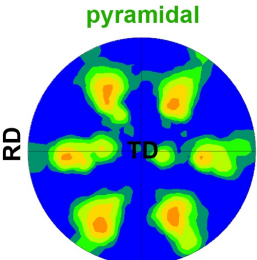

Texture: IPF RD Reference

$10 \overline{1} 0$

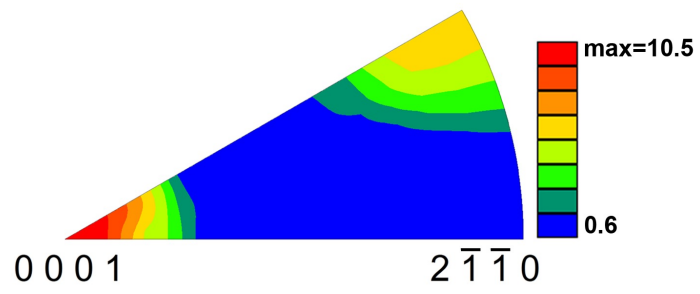

Figure 3: DIC measurements were collected from the microstructural region shown in the IPF map, loaded uniaxially in tension along the horizontal direction, which is also the sample rolling direction. The vertical direction is the sample normal direction. The pole figures (PF) and the IPF texture plot indicate strong [0001] and moderate [1010] textures. These texture components are evident as the red and blue regions in the microtextured regions (MTRs) shown in the IPF maps. Three specific MTRs were studied, one having predominant orientation near the [1010] (labeled as 1 ), another having orientation near the [0001] (labeled as 2), and a region with a more random orientation distribution (labeled as 3 ).
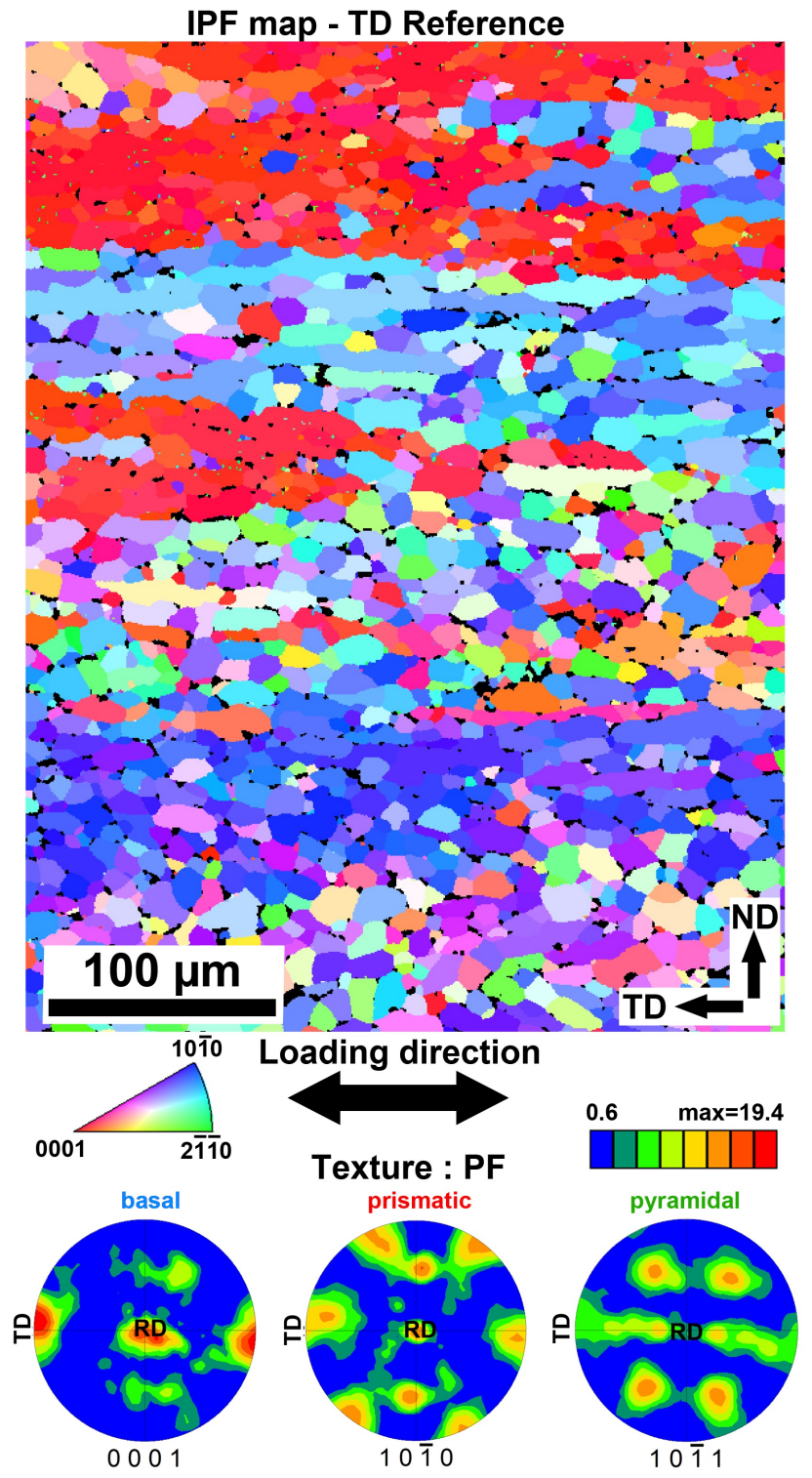

Texture: IPF TD Reference

$10 \overline{1} 0$

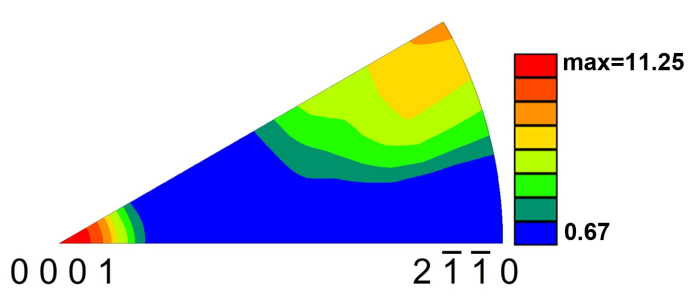

Figure 4: DIC measurements were collected from the microstructural region shown in the IPF map, loaded uniaxially in tension along the horizontal direction, which is also the sample transverse rolling direction. The vertical direction is the sample normal direction. The pole figures $(\mathrm{PF})$ and the IPF texture plot indicate strong [0001] and moderate [1010] textures. These texture components are evident as the red and blue regions in the microtextured regions (MTRs) shown in the IPF maps. The texture between the RD-ND and TD-ND oriented EBSD scans are similar, with a small difference in [0001] and [1010] peak intensity. 


\section{Sub-grain strain map (HR DIC - in-situ tension)}

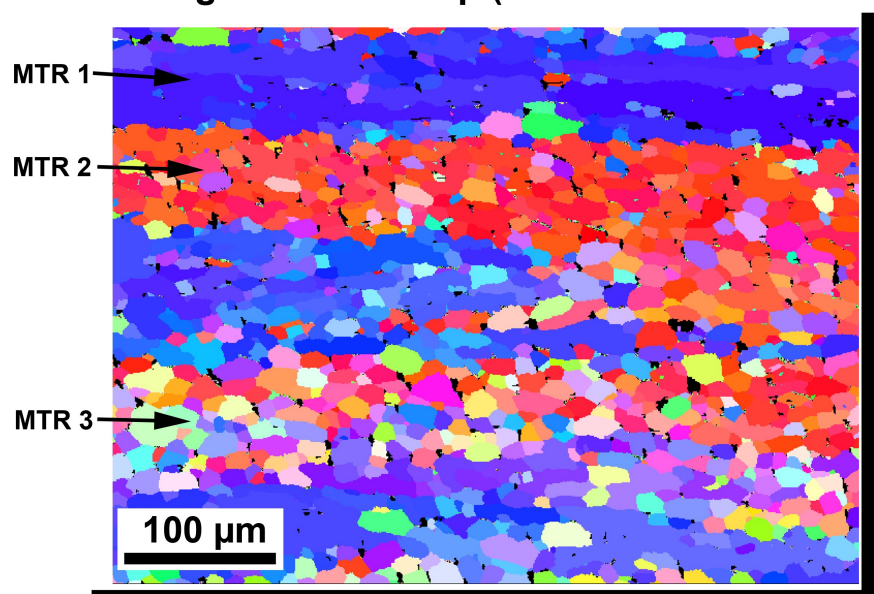

$0.65 \%$ macro strain
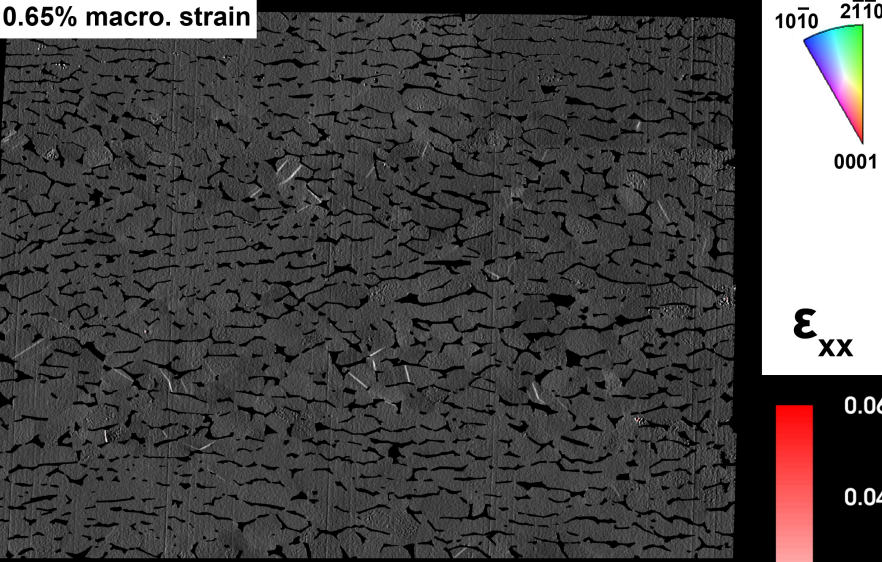

$\varepsilon_{x x}$
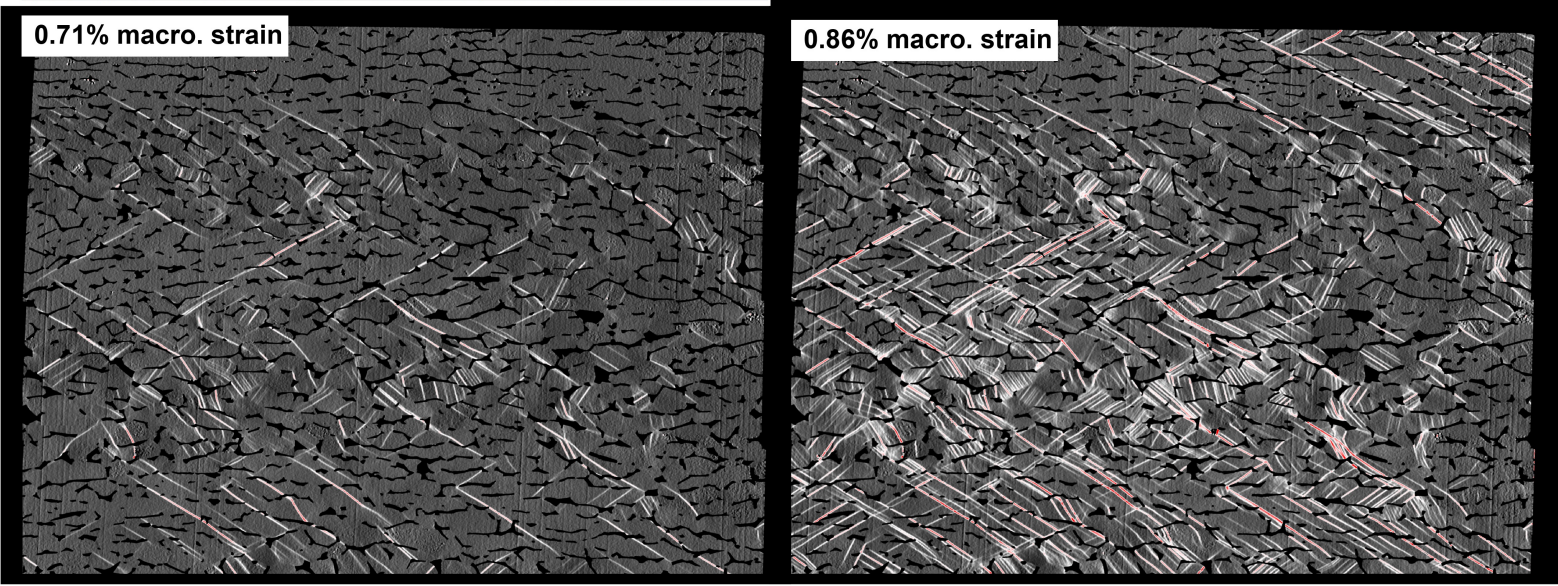

Figure 5: Strain maps measured below macroscopic yielding using SEM DIC are shown in relation to the microstructure and grain orientations (IPF map) measured using EBSD for $0.65 \%, 0.71 \%$ and $0.86 \%$ strain. The sample rolling direction (RD) and the applied tensile loading direction are both horizontal to the page and the sample normal direction (ND) is vertical to the page. 


\section{Sub-grain strain map (HR DIC - in-situ tension)}
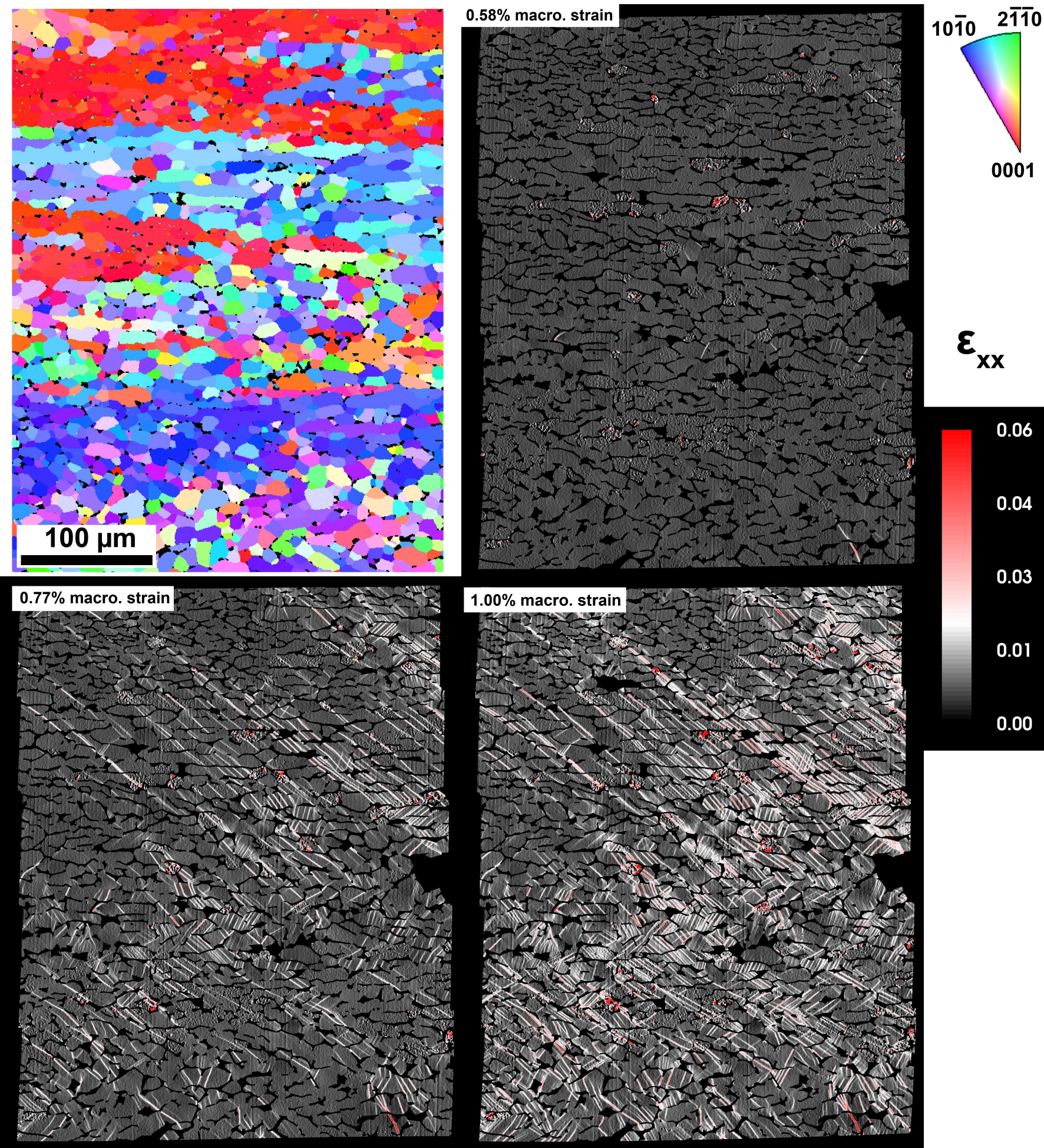

$\varepsilon_{x x}$

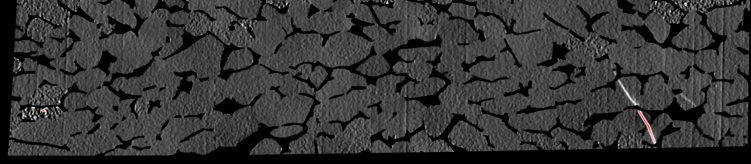

Figure 6: Strain maps measured below macroscopic yielding using SEM DIC are shown in relation to the microstructure and grain orientations (IPF map) measured using EBSD for $0.58 \%, 0.77 \%$ and $1.00 \%$ strain. The sample transverse rolling direction (TD) and the applied tensile loading direction are both horizontal to the page and the sample normal direction (ND) is vertical to the page. 


\section{(a) IPF LD}

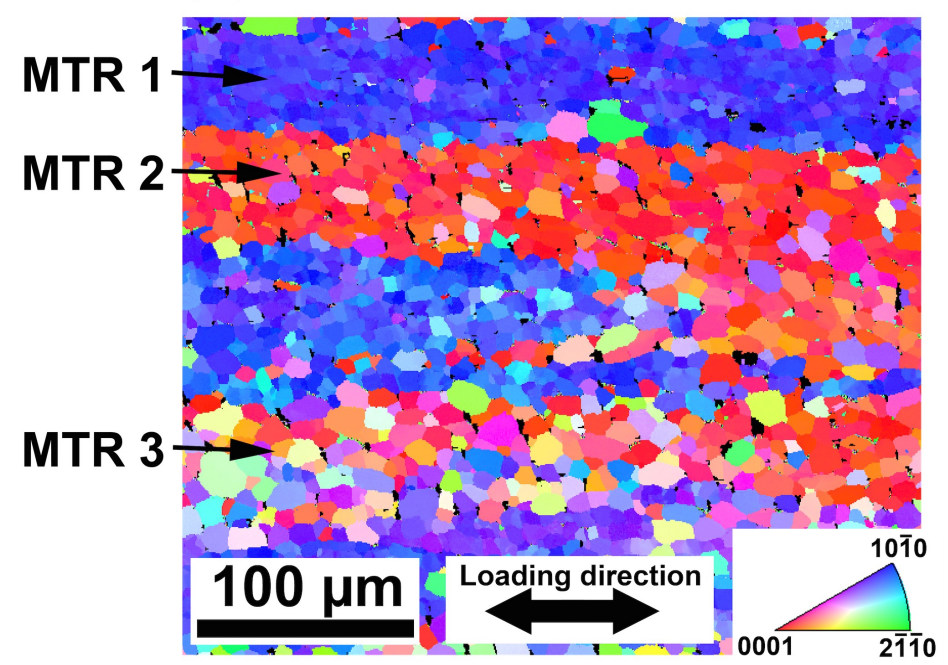

(b) $\varepsilon_{x y}-0.86 \%$ macro. strain

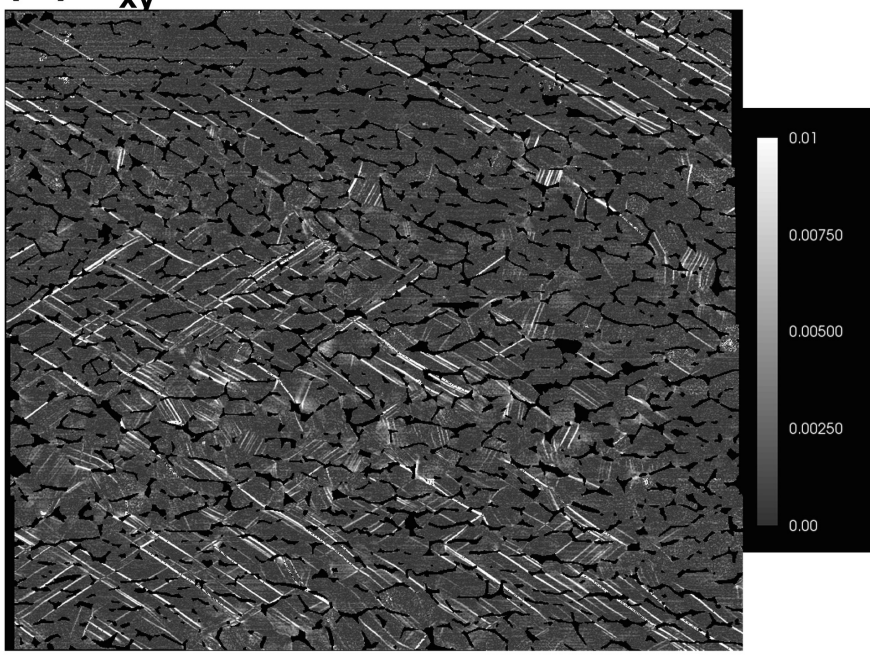

Figure 7: (a) An IPF map referenced to the RD with horizontal loading direction. (b) The absolute value of the measured $\epsilon_{x y}$ shear strain map at $0.86 \%$ macroscopic strain for the same region as in (a).

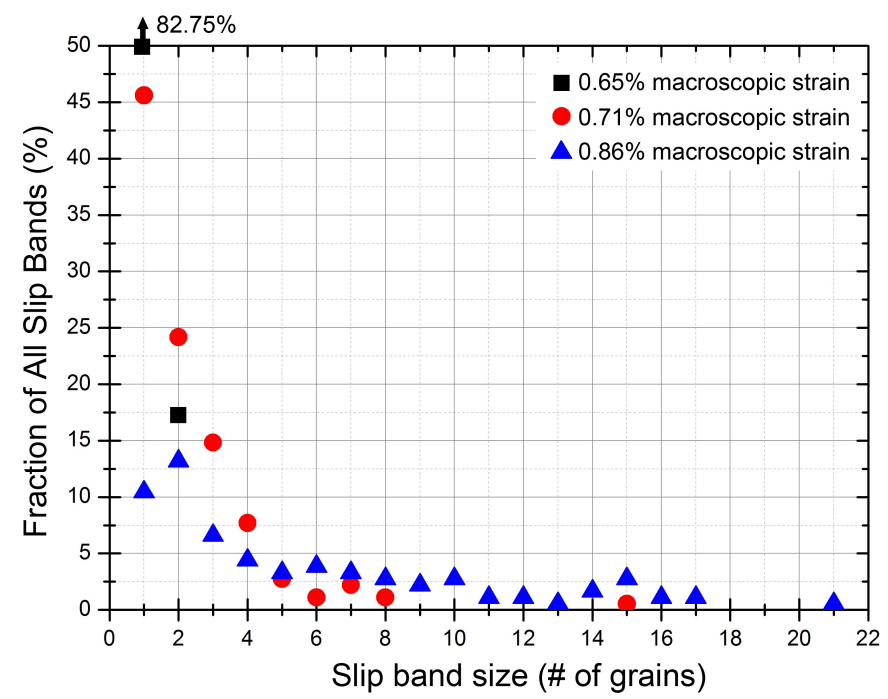

Figure 8: The fraction of total slip bands having localized slip band lengths measured using SEM DIC from the Ti-6Al-4V material imaged in the RD-ND plane, is shown in Figure 5 for each macroscopic strain state. At $0.65 \%$ strain, almost all bands are contained within 1-2 grains. At elevated applied strain, the slip band localization band length extends to as many as 21 grains. All measurements are still well below the macroscopic yield stress of the material, indicating that long ranging plasticity occurs even before the onset of macroscopic plasticity. 
(a) before straining

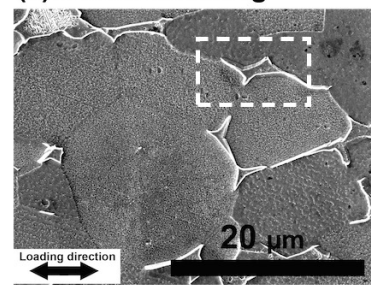

(c) $0.58 \%$ macro. strain

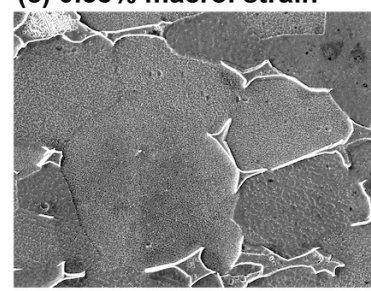

(e) $0.77 \%$ macro. strain

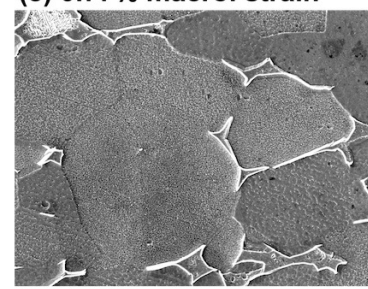

(g) $1.00 \%$ macro. strain

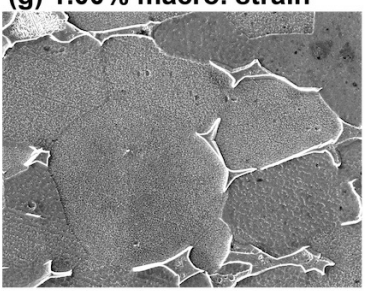

\section{(i) $4.80 \%$ macro. strain}

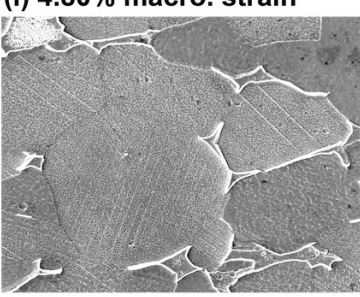

\section{(b) EBSD}

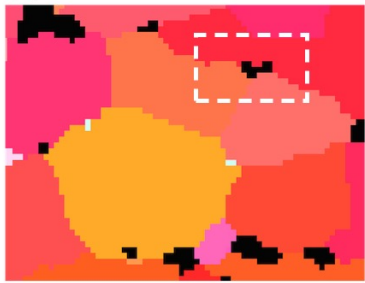

(d) $0.58 \%$ macro. strain

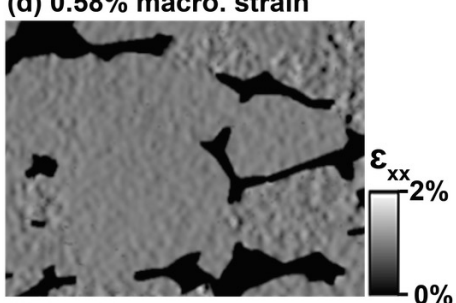

(f) $0.77 \%$ macro. strain

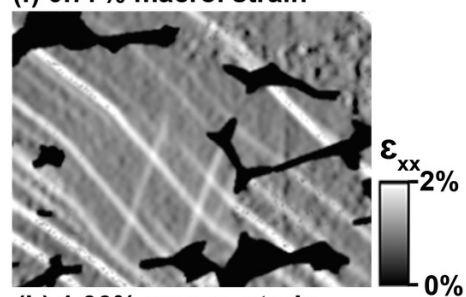

(h) $1.00 \%$ macro. strain

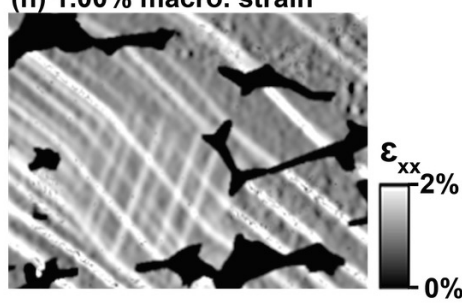

Figure 9: A selected field of view from the SEM DIC experiment from Figure 6 is shown in more detail, including the unloaded microstructure imaged by high resolution secondary electron SEM imaging (a) and the EBSD orientation information (b). With increasing macroscopic strain, local DIC strain measurements show bands extending across $\alpha$ phase grains, through neighboring $\beta$ grains, and into the adjacent $\alpha$ grains (d,f,h). Slip band localization is not visible in any of the secondary electron images (c,e,g) until $4.8 \%$ macroscopic applied strain (i). 


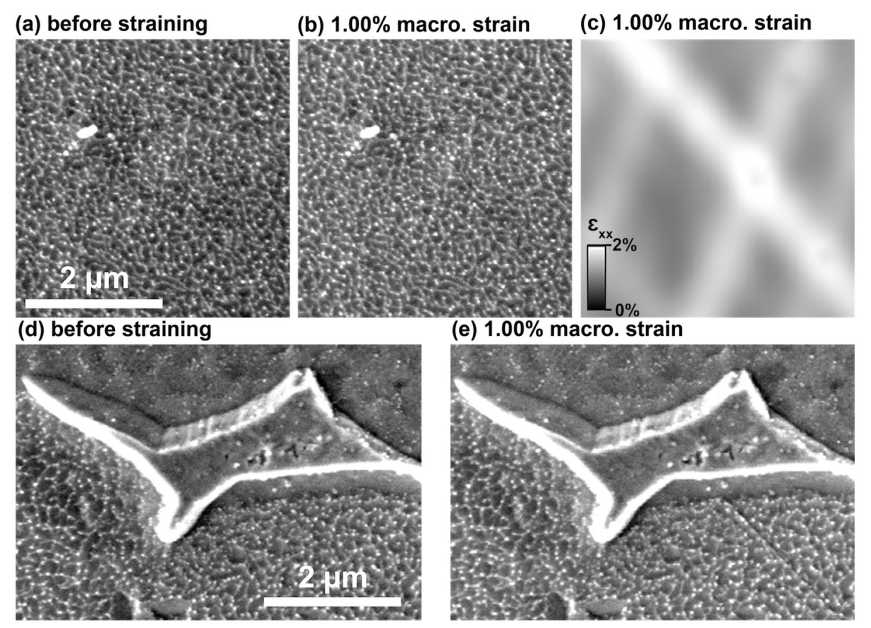

Figure 10: The etched speckle pattern is shown in an $\alpha$ grain before (a) and after (b) $1.00 \%$ macroscopic tensile strain. The measured strain field is shown in (c) for the same region in $(\mathrm{a}, \mathrm{b})$. The displacements resulting in the strain measurement in (c) do not produce visible displacements or slip bands in the high resolution SEM images from (a,b). The field of view shown in the dashed box in Figure 9 is shown in more detail in (d) before straining and (e) after 1.00\% macroscopic strain. A slip band is visible to extend through the $\beta$ phase into the adjacent grain, as stipulated by the strain localization which passes through the region in Figure $9(\mathrm{f}, \mathrm{h})$. 


\section{Slip system activity}
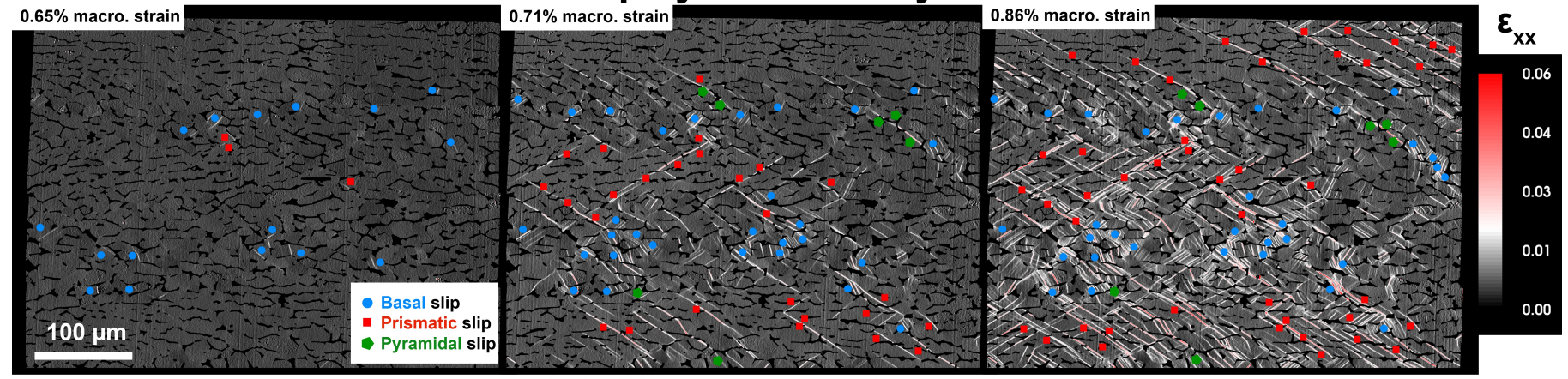

\section{$0.65 \%$ macroscopic strain}
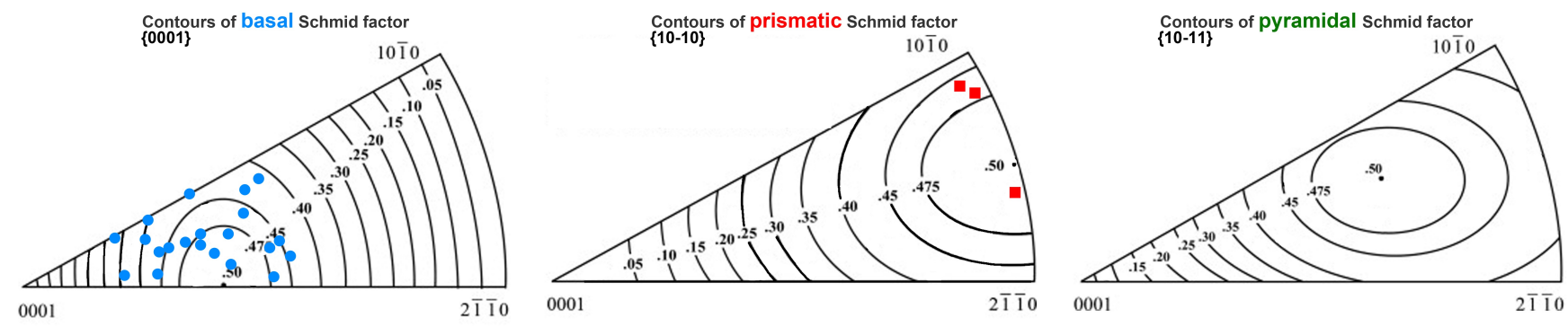

\section{$0.71 \%$ macroscopic strain}
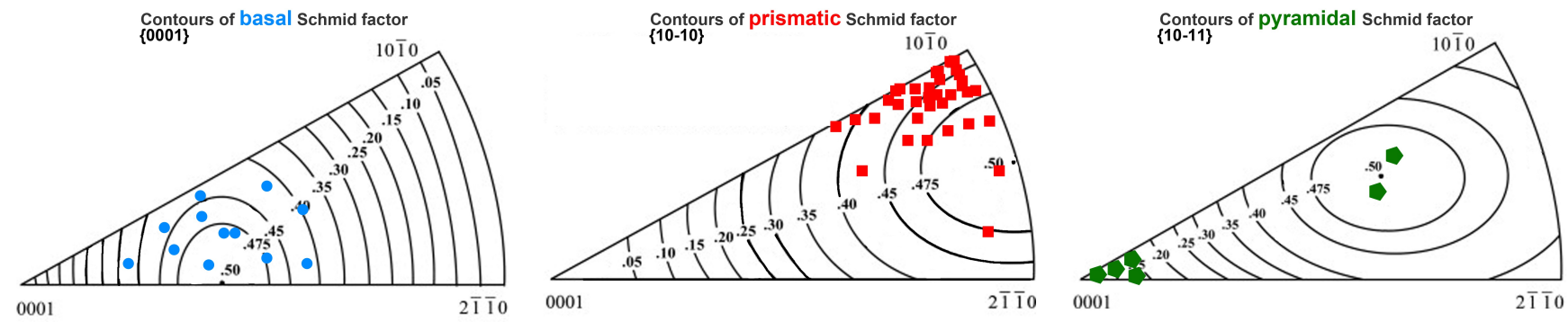

Figure 11: The type of slip system activity is indicated for basal (blue), prismatic (red), and $\langle a\rangle$ pyramidal (green) in the strain maps. The slip type was determined by comparing the calculated surface slip trace from the EBSD orientation data to the observed localization in the DIC measurements. The orientations of grains that show new strain localization since the previous strain increment are plotted on the IPF over the SF contours, in a non-cumulative approach. The loading direction is horizontal to the page, along the sample RD. 


\section{Slip system activity}
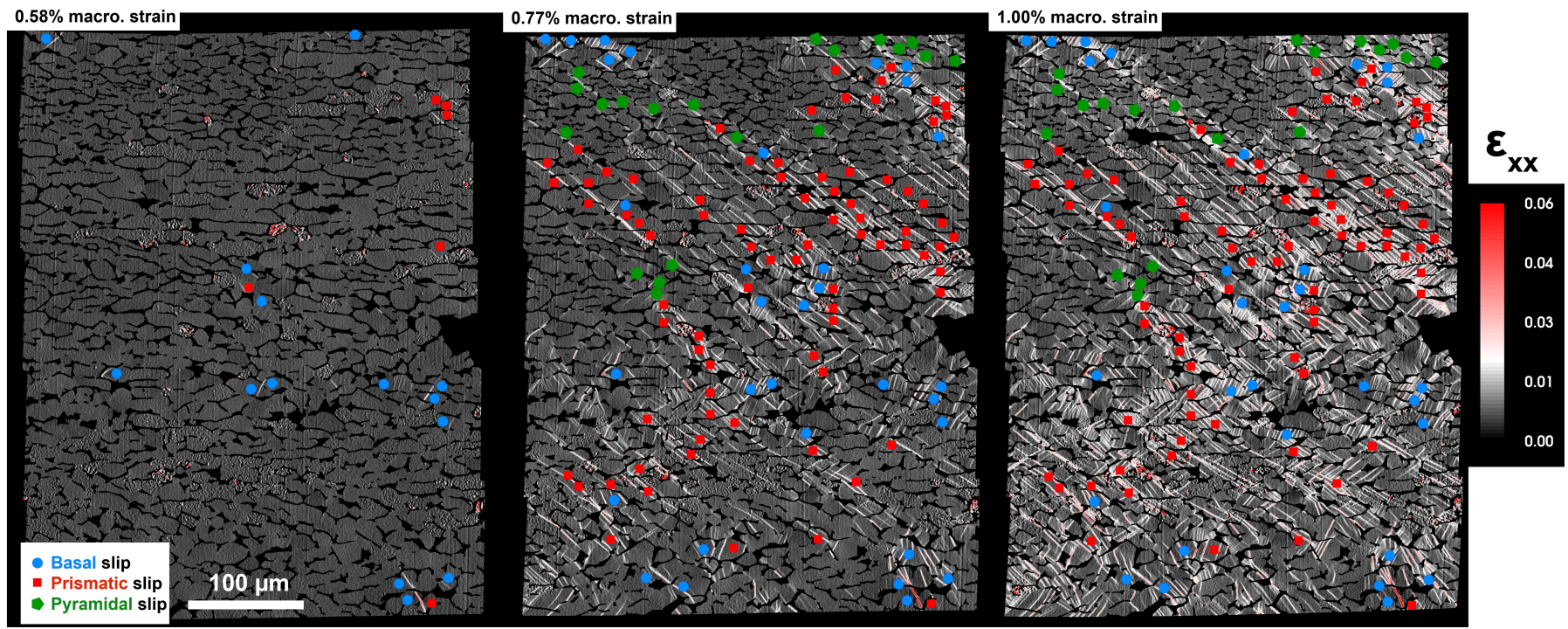

\section{$0.55 \%$ macroscopic strain}
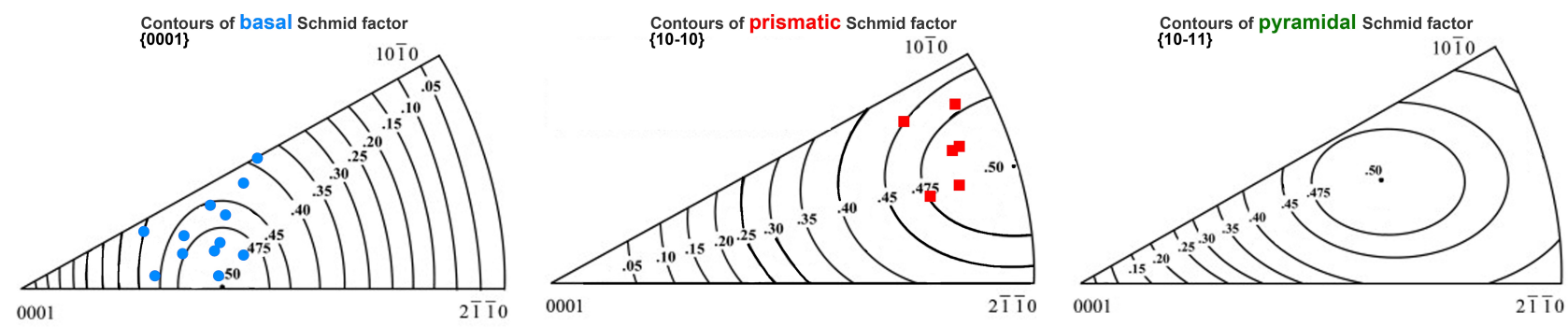

\section{$0.77 \%$ macroscopic strain}
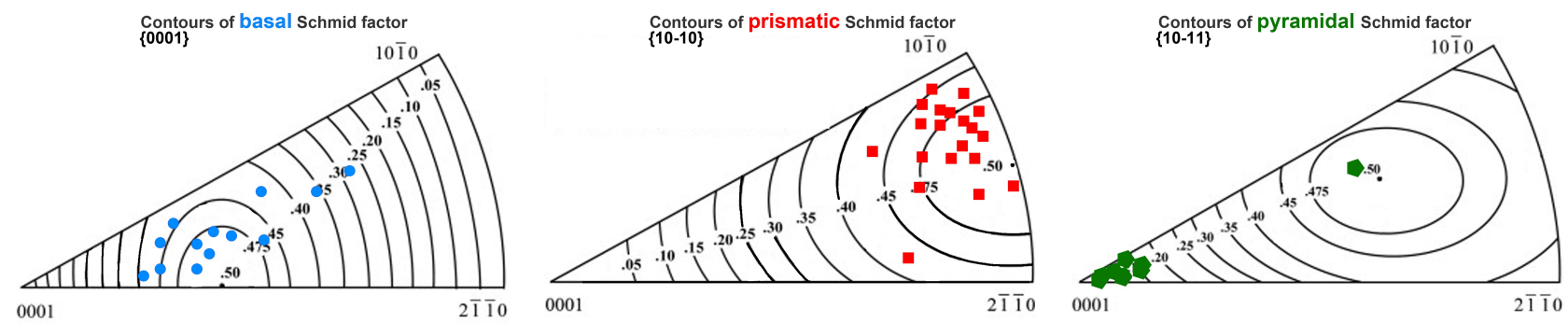

Figure 12: The type of slip system activity is indicated for basal (blue), prismatic (red), and $\langle a\rangle$ pyramidal (green) in the strain maps. The slip type was determined by comparing the calculated surface slip trace from the EBSD orientation data to the observed localization in the DIC measurements. The orientations of grains that show new strain localization since the previous strain increment are plotted on the IPF over the SF contours, in a non-cumulative approach. The loading direction is horizontal to the page, along the sample TD. 
(a)

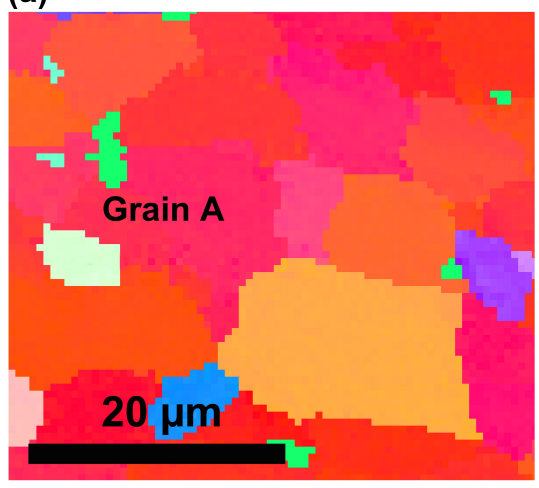

(b)

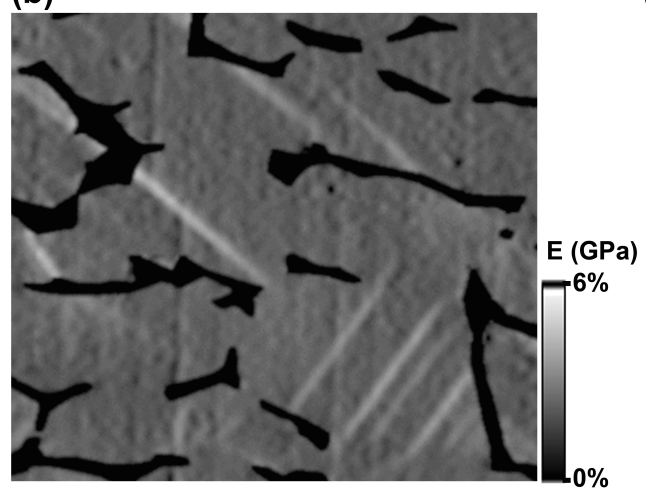

(c)

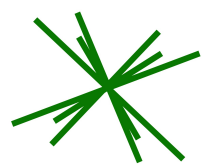

Basal slip trace

max. Schmid factor : $<0.1$

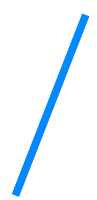

Prismatic slip trace max. Schmid factor : $<0.1$

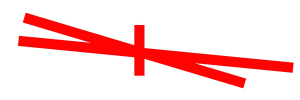

Pyramidal slip trace $\{10 \overline{1} 1\}$

max. Schmid factor $<a>:<0.2$

max. Schmid factor $<a+c>1$ st order $:>0.45$

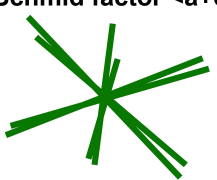

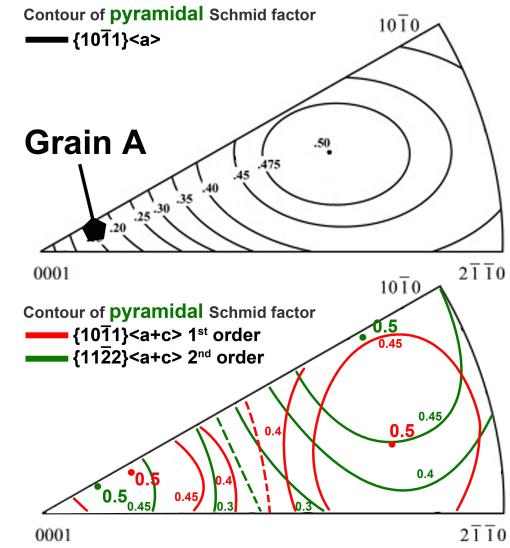

Pyramidal slip trace $\{11 \overline{2} 2\}$

max. Schmid factor $<a+c>2^{\text {nd }}$ order $:>0.45$ 


\section{Titanium 6Al - 4V Alloy}
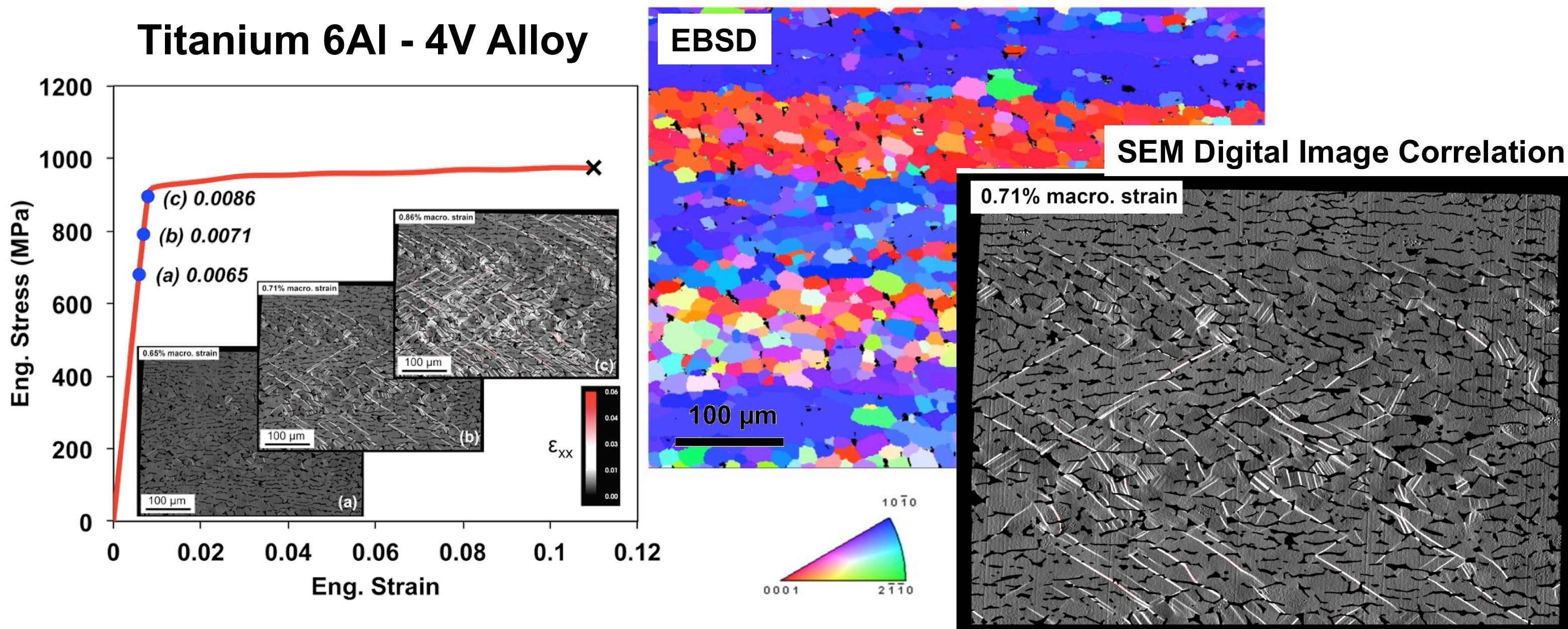